\title{
Forschungen des Kaiser-Wilhelm-Instituts für Arbeitsphysiologie im Nationalsozialismus
}

Das Kaiser-Wilhelm-Institut (KWI) für Arbeitsphysiologie wurde Ende 1912 gegründet und nahm Anfang 1913 zunächst in Berlin unter dem Physiologen und Hygieniker Max Rubner (1854-1932), Professor an der Berliner Universität, seine Arbeit auf. Unter seinem Nachfolger, dem Mediziner/Physiologen Prof. Dr. Edgar Atzler (1887-1938), wechselte das Institut Ende 1929 nach Dortmund ins Ruhrgebiet. In Münster wurde noch eine Zweigstelle unter der Leitung von Prof. Dr. Erich Albert Müller (1898-1977) eingerichtet, die bereits Anfang 1941 ihre Tätigkeit einstellte. An den Ergebnissen der Forschung waren nicht nur die Industrie, sondern auch das Militär und der Sport interessiert. Vor allem für das Militär erwiesen sie sich als „eminent wichtig“.

Das KWI entwickelte sich nach dem Zweiten Weltkrieg - nun als Max-PlanckInstitut - unter der Leitung des Mediziners/Physiologen Prof. Dr. Gunther Lehmann (1897-1974) zum institutionellen Zentrum der Arbeitswissenschaft, was sich schon darin zeigt, dass sich die entsprechenden Hochschullehrer der fünfziger bis achtziger Jahre ganz überwiegend aus dem wissenschaftlichen Personal dieses Instituts rekrutierten. Heute ist es nicht mehr Mitglied der Max-PlanckGesellschaft (MPG). Unbenannt wurde es 2009 in Leibniz-Institut für Arbeitsforschung an der Technische Universität Dortmund. ${ }^{2}$ Gut möglich, dass diese institutionelle Veränderung auch dem Strukturwandel im Ruhrgebiet geschuldet ist. Schwere körperliche Arbeit im Bereich von Bergbau und Eisen/Stahl war nämlich in der Vergangenheit ein wichtiger Aspekt des Forschungsprofils.

Die Entwicklung der Arbeitswissenschaft als eine angewandte und mithin eine dem Anspruch nach interdisziplinäre Wissenschaft steht im Zusammenhang mit der in den zwanziger Jahren des vergangenen Jahrhunderts von Vertretern maßgeblicher Verbände und Wissenschaftsdisziplinen geführten Diskussion über Rationalisierung bzw. Rationalisierung der Arbeit. Unter dem Dach der

\footnotetext{
1 Rüdiger Hachtmann, Ein Kind der Ruhrindustrie? Die Geschichte des Kaiser-WilhelmInstituts für Arbeitsphysiologie von 1913-1945, in: WestfF 60 (2010), S. 73-154, hier S. 132.

2 Zeittafel zur Geschichte des KWI für Arbeitsphysiologie, in: Theo Plesser/Hans-Ulrich Thamer (Hrsg.), Arbeit, Leistung und Ernährung. Vom Kaiser-Wilhelm-Institut für Arbeitsphysiologie in Berlin zum Max-Plank-Institut für molekulare Physiologie und Leibniz Institut für Arbeitsforschung in Dortmund, Stuttgart 2012, S. 25 f.
} 
Arbeitswissenschaft versammelten sich hierzu Vertreter der Natur-, Ingenieursowie der Wirtschafts- und Sozialwissenschaften. Schon 1920 wurde unter dem vorgesehenen Leiter Max Weber (1864-1920), der allerdings noch im gleichen Jahr starb, der Reichsausschuss für Arbeitswissenschaft gegründet, der ein interdisziplinäres Forschungsprogramm voran bringen sollte. Der Ausschuss scheiterte kurz nach der Einberufung am Widerstand der Industrie. ${ }^{3}$ Zur Interdisziplinarität hatte Weber im Rahmen der Forschungen des Vereins für Socialpolitik bereits wichtige methodologische Grundlagen gelegt. ${ }^{4}$ Zudem hatte er bis heute aktuelle Ausführungen zum Problem der Wertfreiheit und zur Objektivität in der (Sozial)Wissenschaft gemacht, die uns im weiteren Verlauf noch beschäftigen werden. ${ }^{5}$ In den arbeitswissenschaftlichen Konzepten, die in der Weimarer Republik entwickelt wurden - vornehmlich von Psychologen wie Otto Lipmann (1880-1933), Paul Plaut (1894-1960) und Fritz Giese (1890-1935) - ist der wissenschaftliche Einfluss Webers offensichtlich. ${ }^{6}$ Diese so genannte Versozialwissenschaftlichung - eine durchaus gängige Tendenz - bedeutet zugleich, dass die Soziologie als Disziplin in der Regel explizit keine Rolle spielt. ${ }^{7}$

Technik und Arbeitsorganisation sind wichtige Ansatzpunkte der Rationalisierung. Insofern kann nicht erstaunen, dass Frederick Winslow Taylor (1856-1915) mit seinem System der Wissenschaftlichen Betriebsführung als ein wichtiger, aber umstrittener Gründer der Arbeitswissenschaft gilt. ${ }^{8}$ Ein zentraler Vorwurf lautet, Taylor orientiere sich letztlich an Maximalleistungen und betreibe dadurch Raubbau am Menschen. Es handele sich daher um eine „Fehlrationalisierung“. 9 In der damaligen Diskussion tauchten unterschiedliche

3 Irene Raehlmann, Interdisziplinäre Arbeitswissenschaft in der Weimarer Republik. Eine wissenschaftssoziologische Analyse, Opladen 1988, S. 50ff.

4 Max Weber, Methodologische Einleitung für die Erhebungen des Vereins für Socialpolitik über Auslese und Anpassung (Berufswahlen und Berufschicksal) der Arbeiterschaft in der geschlossenen Großindustrie (1908), in: ders., Gesammelte Aufsätze zur Soziologie und Sozialpolitik, Tübingen 1924, S. 1-60; ders., Zur Psychophysik der industriellen Arbeit (1908/9), in: ebd. S. 61-255.

5 Max Weber, Die „Objektivität“ sozialwissenschaftlicher Erkenntnis, in: ders., Soziologie, Weltgeschichtliche Analysen, Politik, 3. durchg. und erg. Aufl., Stuttgart 1964, S. 186ff.; ders., Der Sinn der „Wertfreiheit“ der Sozialwissenschaften, in: ebd., S. $263 f f$.

6 Raehlmann, Arbeitswissenschaft Weimarer Republik, S. 88ff.

7 Irene Raehlmann, Arbeitswissenschaft und Soziologie - eine produktive Beziehung?, in: Soziologie 3 (2009), S. 307-315.

8 Frederick Winslow Taylor, Die Grundsätze wissenschaftlicher Betriebsführung, München/ Berlin 1919.

9 Otto Bauer, Kapitalismus und Sozialismus nach dem Weltkrieg, Bd. I.: Rationalisierung Fehlrationalisierung, Berlin 1931. 
Begriffe auf wie physiologische Rationalisierung, psychoenergetisches Optimum, Optimalisierung, Arbeitsbestgestaltung. Sie avancierten zum Leitbild arbeitswissenschaftlicher Forschung und Gestaltung, etwa vergleichbar mit dem heute üblichen Zielkonzept Humanisierung der Arbeit, menschengerechtere Arbeitsgestaltung, gute Arbeit, dass auch das von der Sozial-liberalen Koalition (1969-1982) Anfang der siebziger Jahre initiierte Forschungs- und Aktionsprogramm „Humanisierung der Arbeit“ programmatisch anleitete. ${ }^{10}$ Gleichwohl waren sich die ArbeitswissenschaftlerInnen trotz anders lautender Rhetorik in der Mehrzahl stets bewusst, dass die betriebliche Praxis eher Maximal- denn Optimalleistungen anstrebt. Es geht ihr stets um Kosten- und nicht um Kraftersparnis. WissenschaftlerInnen können nicht damit rechnen, dass ihre Vorschläge zur Arbeitsgestaltung unmittelbar umgesetzt werden. Vielmehr sind diesezumal seit der Novellierung Betriebsverfassungsgesetzes 1972 (vgl. §§ 90, 91) Gegenstand von Verhandlungen zwischen Management und Betriebsrat. Das bedeutet, dass Verbesserungen der Arbeitsbedingungen mit einer Verschlechterung einhergehen können. Beispielweise kann eine ergonomische Verbesserung zu einer Arbeitsintensivierung führen. Die diesbezüglichen Erfahrungen wissenschaftlicher ExpertInnen sind daher durchaus ernüchternd, denn ihr Einfluss ist eher indirekt. Das gilt ebenfalls für die NS-Zeit. Von einer unmittelbaren, direkten Umsetzung kann auch hier nicht ausgegangen werden.

Die Forschungen in der NS-Zeit bedeuteten vielfach keinen grundlegenden Neuanfang, sondern weisen durchaus Kontinuität zur bisherigen und auch späteren Forschung auf. Die Radikalisierung während dieser Zeit bestand etwa darin, dass bisherige normative Begrenzungen offiziell, häufig auch inoffiziell außer Kraft gesetzt wurden. Die wissenschaftliche Neugier und der Ehrgeiz der Forscher waren allem Anschein nach allemal größer, als es ihre eventuell noch vorhandenen moralischen Skrupel waren. Allerdings führten erst die politischen und gesellschaftlichen Rahmenbedingungen zur normativen Entgrenzung. Das gilt auch für die Arbeitswissenschaft. Mit der Zerschlagung der Gewerkschaften, der Abschaffung des Betriebsrätegesetzes und der Betriebsräte wurde in den Unternehmen die alte, patriarchalisch-autoritäre Ordnung wieder hergestellt. Das Management konnte nun widerspruchslos Forschungen erlauben. Dazu gaben in der Regel auch politische Akteure ihr Plazet. Diese waren gegebenenfalls in der Lage, finanzielle Zuwendungen an die Wissenschaftler, also Drittmittelforschung $\mathrm{zu}$ ermöglichen. Als eine weitere Voraussetzung gilt, dass infolge des rassistisch und imperialistisch motivierten Krieges ausreichendes „Men-

10 Irene Raehlmann, Innovationen in Arbeits- und Alltagswelt. Voraussetzung - Wirkungen Barrieren, Göttingen 2007, S. 68ff. 
schenmaterial“ in Gestalt von KZ-Häftlingen, Kriegsgefangenen und sonstigen ZwangsarbeiterInnen als „Untersuchungsobjekt“ zur Verfügung stand.

Meine Untersuchung zur Geschichte des Instituts im Nationalsozialismus verfolgte zwei Perspektiven, die miteinander verbunden sind bzw. in Wechselwirkung stehen. ${ }^{11}$ Da Wissenschaft und das Handeln ihrer Akteure nicht autonom sind, sondern von politischen, gesellschaftlichen und ökonomischen Rahmenbedingungen mitbestimmt werden, sind diese Kontexte $\mathrm{zu}$ berücksichtigen. Die Institutionen der Wissenschaft und das Handeln von WissenschaftlerInnen sind dadurch aber keineswegs voll determiniert. Wissenschaft verfügt über eine Eigendynamik und WissenschaftlerInnen verfügen über eine begrenzte Autonomie in ihrem allgemeinen sozialen und auch in ihrem speziellen, Erkenntnis produzierenden Handeln. Diese grundlegenden Annahmen führten mich zu zwei Untersuchungsebenen: Erstens galt es die institutionellen Rahmenbedingungen in den Blick zu nehmen. Zweitens war auch das Handeln der Akteure als Wissenschaftler und auch allgemeiner als Mitglied des wissenschaftlichen und politisch-gesellschaftlichen Systems $\mathrm{zu}$ untersuchen. Beide Untersuchungsebenen werden von den angesprochnen Rahmenbedingungen beeinflusst bzw. bilden damit Wirkungszusammenhänge. Das Handeln der Akteure wird zudem von dem arbeitsorganisatorischen Kontext mit geprägt.

\section{Die Arbeitsbereiche des KWI für Arbeitsphysiologie}

Das KWI für Arbeitsphysiologie beschäftigte sich im Nationalsozialismus vor allem mit zwei Forschungsvorhaben: Der Ernährungsforschung sowie der noch stärker kriegswirtschaftlich ausgerichtete Bereich um den Gasschutz. Die beiden Bereiche der Institutsforschung haben auf den ersten Blick nichts Gemeinsames. Eine Klammer bildet jedoch die Erforschung der physiologischen und gegebenenfalls auch der psychologischen Voraussetzungen menschlicher Leistung und deren Grenzen. Obwohl es sich gemäß dem Selbstverständnis der Wissenschaftler um Grundlagenforschung handelt, verbindet sich diese mit einem Praxisbezug. Es ging einerseits um Industriearbeit, vornehmlich um eine ausreichende Ernährung bei schwerer körperlicher Arbeit, andererseits um militärisch wichtige Tätigkeiten wie das Kämpfen unter Schutzkleidung, etwa Gasmasken, und

11 Irene Raehlmann, Arbeitswissenschaft im Nationalsozialismus. Eine wissenschaftssoziologische Analyse, Wiesbaden 2005. 
um die ergonomisch optimale Konstruktion von Waffen und deren Gebrauch. Arbeit, die im Verständnis der Moderne durchweg positiv wahrgenommen und überaus wertgeschätzt wird, erhält durch die NS-Ideologie einen weiteren, diesmal allerdings negativen Sinngehalt. Es erfolgt eine Militarisierung von Arbeit, demzufolge das Töten im Krieg, aber auch der Genozid als Arbeit bezeichnet werden. Die Tätigkeiten werden heute dem Bereich „destruktive(r) Arbeit“ zugeordnet. ${ }^{12}$ Diese Bezeichnung wird sich auch für die Ernährungsforschung als berechtigt erweisen. Im Ersten Weltkrieg hatte sich das Thema Ernährung und Leistung zu einem wichtigen Forschungsfeld entwickelt. Atzler knüpfte nach einigen Jahren der Unterbrechung in Folge der militärischen Niederlage im Ersten Weltkrieg an die von Rubner begründete Rüstungsforschung - zunächst im Geheimen - wieder an. Für die beiden Forschungsfelder empfing das Institut über Jahre umfangreiche Geldmittel aus den zuständigen Ministerien.

Der dritte Bereich Arbeitsbereich des KWI für Arbeitsphysiologie war der Sport. Erkenntnisse der Sportphysiologie sollten für die Gestaltung industrieller Arbeit genutzt und sportliche Aktivitäten als Ausgleich für einseitige körperliche Belastungen gefördert werden. ${ }^{13}$

Während in Dortmund Fragen der Industrieforschung den Schwerpunkt bildeten, waren es in Münster wehrphysiologische und ergonomische Auftragsstudien. ${ }^{14}$ Die Stadt Münster war als Ort einer Zweigstelle gewählt worden, weil sie Standort einer Universität und einer Garnison war. So konnte wissenschaftliches Personal leicht rekrutiert werden. Außerdem „eröffneten sich für wehrphysiologische Großforschungsprojekte Kooperationsperspektiven mit den 2.200 bis 2.300 [...] stationierten Soldaten der Reichswehr“. ${ }^{15}$ Die sich anbahnende Kooperation zwischen Reichswehr und KWI für Arbeitsphysiologie wurde von der Kaiser-Wilhelm-Gesellschaft (KWG) auf Grund beidseitiger Interessen akzeptiert. War doch damit zugleich dem „Gesamtinteresse des Staates“ gedient. Der Präsident der KWG, Adolf von Harnack, begrüßte den darüber hinaus gehenden Plan, geeignete KWG Institute der Wehrmacht „dienstbar zu machen“. Institutionell zeigte sich diese Zusammenarbeit darin, dass einerseits Vertreter des Reichswehrministeriums seit Mitte der zwanziger Jahre Mitglieder im Verwaltungsrat des KWI waren ${ }^{16}$ und andererseits Atzler Mitglied im Psycho-

12 Lars Clausen, Produktive Arbeit, destruktive Arbeit. Soziologische Grundlagen, Berlin/New York 1988.

13 Hachtmann, Kind, S. 87.

14 Florian Schmaltz, Kampfstoff-Forschung im Nationalsozialismus. Zur Kooperation von Kaiser-Wilhelm-Instituten, Militär und Industrie, Göttingen 2005, S. 207.

15 Ebd., S. 204.

16 Ebd., S. 201. 
logischen Senat des Ministeriums wurde. ${ }^{17}$ Atzler, so Schmaltz, „förderte die Militarisierung der physiologischen Forschung im Kontext der getarnten Aufrüstung der Reichswehr in der Weimarer Republik an seinem Institut". ${ }^{18}$ So schlug er einen Forschungskatalog mit ergonomischer und physiologischer Akzentuierung vor, so zum Schnellladegeschütz und Maschinengewehr, zur Normierung von Handgranaten und zur optimalen Marschgeschwindigkeit. ${ }^{19}$ Zwischen 1925 und 1935 kam es zu einer größeren Zahl wehrpolitisch relevanter Forschungsprojekte, die staatlicherseits finanziert wurden. ${ }^{20}$

\section{Die Gasschutzforschung}

Die Gasschutz- bzw. Gasmaskenforschung unter Einbeziehung von Soldaten bildete einen Schwerpunkt dieser Institutsarbeit. Die „Dauertrageversuche mit der Gasmaske“ fanden in mehreren Zyklen zwischen 1932 und 1934 statt. Die Versuchspersonen stammten aus allen Formationen des Heeres, aber eine Einwilligung zur Teilnahme hatten sie nicht gegeben. Ihre Teilnahme wurde angeordnet, obwohl sie im Winter 1933 noch mit einer Bronchitis zu kämpfen hatten, was das Atmen unter einer Gasmaske erschwerte. Diese Beeinträchtigungen wurden schlicht ignoriert, um, so die Behauptung, das Forschungsprojekt nicht zu gefährden. Diese Position widersprach jedoch den vom Reichsministerium des Inneren 1931 erlassenen „Richtlinien für neuartige Heilbehandlung und für die Vornahme wissenschaftlicher Versuche am Menschen“. ${ }^{21}$ Dem zu Folge waren wissenschaftliche Untersuchungen am Menschen ohne deren Einwilligung nicht erlaubt. Mögliche Probanden mussten zuvor über Zweck, Begründung, Durchführung und Gefahren des Experiments aufgeklärt werden. Hier zeigt sich, dass bereits vor bzw. gleich am Anfang der NS-Herrschaft normative Standards für Forschungen mit und am Menschen außer Kraft gesetzt wurden. Bekanntlich kam es mit der Dauer des Regimes zu einer massenhaften Missachtung solcher Regeln. Das gilt auch, wie noch zu zeigen ist, für die späteren Ernährungsuntersuchungen des KWI für Arbeitsphysiologie.

Die „Soldaten wurden toxischen Reizgasen ausgesetzt, um den dichten Sitz der Gasmasken zu kontrollieren. Im Freien geschah dies mit Hilfe von Spritzge-

\footnotetext{
17 Ebd., S. 243.

18 Ebd.

19 Ebd., S. 200.

20 Ebd., S. 201.

21 Ebd., S. 221.
} 
räten, mit denen das Manövriergelände überraschend vernebelt wurde““.22 Infolgedessen litten die Männer unter Schmerzen am Kopf, starkem Tränen der Augen und Augenschmerzen sowie Atemnot und Brechreiz, bisweilen kam es auch zu Gasvergiftungen. Die Gase sollten dem Gegner im Krieg das Tragen von Gasmasken unmöglich machen und ihn damit anderer tödlicher Giftgase schutzlos ausliefern. Für die gesundheitlichen Gefährdungen der Teilnehmer interessierten sich jedoch die die Experimente durchführenden Wissenschaftler bestenfalls im Rande. Deren „leistungsmedizinisch orientierte(r) Blick [...] war allein auf die Frage gerichtet, inwiefern das dauerhafte Tragen der Gasmasken zu einer physiologisch meßbaren Leistungsminderung führte“. ${ }^{23}$ Während Atzler für die im Winter organisierten Experimente keine Leistungsminderung behauptete, kam er für die im Sommer durchgeführten Untersuchungen zu einem gegenteiligen Ergebnis. Die beteiligten Wissenschaftler interessierten sich letztlich für Leistungsgrenzen der Gasmasken tragenden Soldaten. Ergänzt wurden die physiologisch orientierten Untersuchungen durch ergonomische, die eine optimale Gestaltung der Gasmaske und ein optimales Zusammenspiel von Gasmaske und Stahlhelm zum Ziel hatten.

\section{Leistungsernährungsforschung}

Das weitere zentrale Forschungsfeld während der NS-Zeit, vor allem während des Krieges war der Zusammenhang zwischen Ernährung und Leistung. ${ }^{24}$ Damit war zeitweilig das gesamte Dortmunder Institut beschäftigt, das dafür seine finanziellen und personellen Ressourcen erheblich aufstocken konnte. Die Leitung dieser Vorhaben hatte der Ernährungsphysiologe Prof. Dr. Heinrich Kraut (1893-1992) inne. ${ }^{25}$ Nach dem Studium, der Promotion und Habilitation in Chemie stand er seit 1928 der chemischen Abteilung des Instituts vor, er wurde 1932 außerplanmäßiger Professor und 1951 Honorarprofessor an der Universität Münster. Er war Mitglied der NSDAP seit 1937. Sein Renommee als international anerkannter Ernährungsexperte führte dazu, dass er seine Karriere bruchlos vom Dritten Reich in die Dritte Welt fortsetzen konnte. Er wurde Präsident der Welthungerhilfe. Diese Organisation stiftete überdies den „Professor-Kraut-

\footnotetext{
22 Ebd., S. 222.

23 Ebd., S. 225.

24 Raehlmann, Arbeitswissenschaft Nationalsozialismus, S. 98ff.

25 Ebd., S. 107; Ulrike Thoms, Das Max-Plank-Institut für Ernährungsphysiologie und die Nachkriegskarriere von Heinrich Kraut, in: Plesser/Thamer, Arbeit, S. 295-356.
} 
Preis“, womit hervorragende Leistungen von Ernährungsberatern aus Entwicklungsländern ausgezeichnet wurden und womöglich noch werden. Kraut bekleidete viele herausragende Funktionen in einschlägigen Organisationen und erhielt zahlreiche Auszeichnungen, die hier nicht alle aufgeführt werden können. So wurde ihm das große Verdienstkreuz des Verdienstordens der Bundesrepublik Deutschland, die Ehrendoktorwürde der Medizinischen Fakultät der Universität Münster und wohl auch die der Universität Be'er Sheva/Israel verliehen. Die zuletzt genannte Ehrung erfolgte vermutlich in Unkenntnis seiner NSDAP-Mitgliedschaft, seiner Forschungen in der NS-Zeit und seiner Gutachten im Rahmen der Nürnberger Prozesse gegen den IG Farben- und den FlickKonzern, in denen er die Ernährungsgrundlagen für die Häftlinge im $\mathrm{KZ}$ Auschwitz als ausreichend bewertet hatte. ${ }^{26}$ Diese steile, ungebrochene, internationale Karriere in der Nachkriegszeit war ja für ehemalige Mitglieder der NS-Elite keineswegs ungewöhnlich.

Das Forschungsfeld Ernährung und Leistung steht ganz in der Tradition des KWI. Wie schon im Ersten Weltkrieg erfuhr dieses Thema in der NS-Zeit einen enormen Bedeutungszuwachs, der auch noch in den Hungerjahren der Nachkriegszeit anhielt. In der späteren Entwicklungspolitik war es ebenfalls zentral. Fragen der Ernährung wurden zu einem erstrangigen Thema, weil die mangelhafte Ernährung während des Ersten Weltkrieges als eine wesentliche Ursache für Kriegsmüdigkeit und die Entstehung einer politischen Opposition angesehen wurde. ${ }^{27}$ Mit Hilfe von Ausbeutung und Vernichtung der Bevölkerung in den eroberten Ländern wurden die Deutschen bis Kriegsende einigermaßen ausreichend ernährt. ${ }^{28}$ Kraut trat schon vor dem Krieg als Herausgeber eines Volkskochbuches für das rheinisch-westfälische Industriegebiet hervor, ferner entwickelte er im Krieg „Rationssätze für die verschiedenen Bevölkerungsgruppen“, die als „Kraut'sche Normen“ für die Nahrungszuteilung maßgebend wurden.

Das Thema Ernährung und Leistung verweist auf grundlegende Annahmen arbeitswissenschaftlicher Forschung. Von Beginn an galten vor allem die physiologischen und psychologischen Faktoren als maßgeblich bestimmend für die Leistung der Arbeitskräfte. Dabei wurde Leistung differenziert betrachtet, nämlich als Leistungsfähigkeit und Leistungsbereitschaft. Diese Unterscheidung

26 Meine diesbezügliche Anfrage in Be'er Sheva blieb ohne Antwort, d. h. die Ehrung wurde weder bestätigt noch verneint.

27 Christian Gerlach, Krieg, Ernährung, Völkermord. Forschungen zur deutschen Vernichtungspolitik im Zweiten Weltkrieg, Hamburg 1989, S. 169 u. 246.

28 Ulrich Herbert, Fremdarbeiter. Politik und Praxis des „Ausländer-Einsatzes“ in der Kriegswirtschaft des Dritten Reiches, 2. Aufl. Berlin/Bonn 1999, S. 431. 
spielt nachfolgend noch eine wichtige Rolle. Das naturwissenschaftliche Profil des KWI umfasste daher auch psychologische Kompetenz.

Offiziell distanzierten sich die Wissenschaftler des KWI von der im Krieg immer stärker werdenden Forderung nach Maximalleistungen und hielten das Prinzip der Optimalleistung hoch. Sie beschworen immer wieder das Prinzip der Wertfreiheit und stilisierten das Institut zu einer „Pflegestätte unabhängiger Forschung“. Gemäß dem programmatischen Leitbild der KWG verstanden auch sie ihre wissenschaftliche Tätigkeit als Grundlagenforschung. Ihr Selbstverständnis, größtenteils Selbsttäuschung, wurde auch in der Nachkriegszeit aufrechterhalten und gepflegt. Die Leistungsforschung bot die Chance, grundlegende Erkenntnisse über die Grenzen menschlicher Leistungsfähigkeit zu gewinnen. Meines Erachtens bestand darin die Attraktivität für die Wissenschaftler. Dieses Interesse ließ sie in beiden Tätigkeitsfeldern normative Grenzen überschreiten.

Die Forschung in Dortmund bezog sich aber nicht nur auf die Ernährung, sondern pharmakologische Substanzen wie Alkohol, Koffein, Nikotin, ferner Phosphat, Lecithin, Colanuss, Vitamine, Traubenzucker und Pervitin sowie Narkotika, etwa Kokain, Morphium, Meskalin, Haschisch und Schlafmittel wurden hinsichtlich ihrer Bedeutung für die Arbeitsleistung und deren Steigerung verstärkt erforscht. Auftraggeber waren häufig pharmazeutische Hersteller. Hinzu kamen betriebliche Untersuchungen, etwa bei Krupp der Einsatz einer Ultraviolett-Bestrahlungsanlage und ein zumindest geplanter Großversuch mit Vitaminen. ${ }^{29}$ Wegen der Risiken war die Zahl der Versuchspersonen jedoch häufig klein und Selbstversuche waren nicht ungewöhnlich. Nahezu durchgängig konstatierten die Forscher nach Einnahme der Substanzen eine Leistungssteigerung, aber sie wiesen auch auf die Gefahr des Dopings hin, d. h. eine momentane Leistungssteigerung schädige bei anhaltendem Gebrauch die Gesundheit und führe überdies letztlich zur Sucht. Schon die andauernden Dopingskandale im Sport heutzutage verweisen auf die Aktualität der Leistungsforschung. Darin sind Sportmediziner - auch aus universitären Instituten - involviert. Der mit Doping einhergehende Raubbau, so die weiterführende Argumentation der KWI-Wissenschaftler, sei aber im Interesse übergeordneter Ziele, um etwa aus dem Krieg als Sieger hervor zu gehen, gerechtfertigt. So wurde beispielsweise Pervitin in allen militärischen Bereichen angewandt. ${ }^{30}$ Dessen Wirkung wird in einem Dokument so beschrieben:

29 Raehlmann, Arbeitswissenschaft Nationalsozialismus, S. 113f.

30 Karl Heinz Roth, Das Beispiel Pervitin, in: Friedolf Kudlien (Hrsg.), Ärzte im Nationalsozialismus, Köln 1985, S. 172. 
Nach Einnahme von 1-5 Tabletten schwinden Schlafbedürfnis und Müdigkeit, statt dessen treten Arbeitslust, Aktivität, Redefluss und Wohlbefinden auf; werden die Tabletten am Abend genommen, so kann man die ganze Nacht ohne Müdigkeit durcharbeiten in voller Leistungsfähigkeit und bei erhaltener Arbeitskraft [...]. Es ist z. B. ganz auffallend, wie stark die Arbeitskraft gerade bei Personen, welche träge und unlustig bei der Arbeit sind, gehoben wird. ${ }^{31}$

In einem anderem Dokument wird über einen Versuch mit Landarbeitern Folgendes berichtet: Unter dem Einfluss von Pervitin arbeitet diese Gruppe regelmäßig 36 Stunden und nach sieben Stunden Schlaf wird wieder 36 Stunden gearbeitet. Die Arbeiter würden nach diesem stimulierenden Mittel verlangen, um durch Überstunden ihren Verdienst zu steigern. ${ }^{32}$

Mit Beginn und im Verlauf des Krieges wurden unter der Federführung von Kraut verschiedene Untersuchungen mit Zwangsarbeitern (russische Kriegsgefangene, Ostarbeiter, italienische Militärinternierte) über die Bedeutung der Kalorienzufuhr für Leistungsfähigkeit und Leistungsbereitschaft durchgeführt. In diesem Zusammenhang waren die Wissenschaftler auch daran interessiert, „rassische Leistungsunterschiede“ aufzudecken. ${ }^{33}$ Es handelte sich, soweit ich sehe, ausschließlich um Männer. Wären Frauen in die Untersuchungen einbezogen worden, so wären vermutlich auch physiologische Unterschiede der Geschlechter thematisiert worden. Die Arbeitskräfte waren auf Grund der Mangelernährung in den Lagern nach wenigen Wochen kaum noch arbeitsfähig. Außer der Hungersnot kam es zu Seuchen, hohen Todesraten, von der systematischen Unterdrückung erst gar nicht zu sprechen. Während der Experimente verbesserte sich zwar die Versorgung, doch generell sollte das Ernährungsgefälle zwischen Deutschen und Zwangsarbeitern aufrechterhalten werden, um Proteste der Bevölkerung nicht aufkommen zu lassen. ${ }^{34}$

Die Naturwissenschaftler in der Arbeitswissenschaft favorisieren Laborexperimente. Hingegen verwiesen die sozialwissenschaftlichen Experten schon früh, so Max Weber, auf die Notwendigkeit von Feldforschung, denn die Ergebnisse der Laborforschung können nicht ohne weiteres auf die reale Arbeitswelt übertragen werden..$^{35}$ Die einmalige Chance zur Feldforschung im großen Stil unter quasi experimentellen Bedingungen bot sich, als das KWI, im Wesent-

31 Archiv zur Geschichte der Max-Planck-Gesellschaft Berlin (künftig: AGMPG) I. Rep. 4, 165, Bl. 1f.

32 Raehlmann, Arbeitswissenschaft Nationalsozialismus, S. 115.

33 Susanne Heim, Kalorien, Kautschuk, Karrieren. Pflanzenzüchtung und landwirtschaftliche Forschung in Kaiser-Wilhelm-Instituten 1933-1945, Göttingen 2003, S. $116 f$.

34 Ebd., S. 114.

35 Raehlmann, Arbeitswissenschaft Weimarer Republik, S. 82f. u. 180. 
lichen finanziert durch das Reichsernährungsministerium, Leistungsuntersuchungen mit 6800 Zwangsarbeitern in zahlreichen Unternehmen des Ruhrgebiets mit so klangvollen Namen wie etwa Krupp und Hoesch durchführen konnte. ${ }^{36}$ Zuvor hatten schon kleinere Untersuchungen stattgefunden. Ein Ergebnis war, dass mit der Einführung von Prämien, etwa Ernährungszulagen und Zigaretten, die Leistung anstieg bei gleichzeitigem Abfall des Körpergewichts. So heißt es in einem Bericht:

[...] unter der Wirkung der durch Prämie forcierten Arbeit (kam, I. R.) es zu einem erheblichen Abfall des Körpergewichtes, so dass eine Fortsetzung dieser Arbeitsweise über längere Dauer unmöglich gewesen wäre. ${ }^{37}$

In einem weiteren Bericht findet sich ein ähnliches Ergebnis:

Seit der Einführung des Zigarettenprämie überstieg dagegen die Leistungsbereitschaft so sehr die Leistungsfähigkeit, dass innerhalb eines halben Jahres 3,5 kg pro Mann zur Deckung des Kaloriendefizits aus den Reserven des Organismus zugesetzt wurden. ${ }^{38}$

Der „Ernährungsgroßversuch“ fand im letzten Kriegsjahr 1944/45 statt, als der militärische Rückzug auf breiter Front einsetzte und sich die Niederlage abzeichnete. „In den besetzten Ländern versteifte sich der Widerstand gegen die wirtschaftliche Ausbeutung und die Deportation von Arbeitskräften“. ${ }^{39}$ Dadurch erhielten die Partisanen erheblichen Zulauf. ${ }^{40}$ Im Zuge dieser Entwicklung erwies sich der zunehmende Arbeitskräftemangel in Deutschland als das zentrale Problem. ${ }^{41}$ Daher mussten alle Leistungsreserven der vorhandenen Arbeitskräfte mobilisiert werden, um so doch noch die Niederlage abwenden zu können. Offizielles Ziel der Ernährungsuntersuchungen war, den schlechten Gesundheitszustand und die ungenügende Leistung der ausländischen Zwangsarbeiter durch eine Erhöhung der Kalorienversorgung, die 90 bis maximal 100 Prozent der Rationen für deutsche Beschäftigte entsprechen sollte, zu verbessern. Kraut ging also davon aus, dass die Arbeitsproduktivität der Zwangsarbeiter dadurch stei-

36 Raehlmann, Arbeitswissenschaft Nationalsozialismus, S. $119 \mathrm{ff}$.

37 AGMPG 1, A1, 1372/4, Bl. 7.

38 Raehlmann, Arbeitswissenschaft Nationalsozialismus, S. 117.

39 Dietrich Eichholtz, Die „Krautaktion“. Ruhrindustrie, Ernährungswissenschaft und Zwangsarbeit 1944, in: Ulrich Herbert (Hrsg.), Europa und der „Reichseinsatz“. Ausländische Zivilarbeiter, Kriegsgefangene und KZ-Häftlinge in Deutschland 1938-1945, Essen 1991, S. 270294, hier S. 270.

40 Heim, Kalorien, S. 111.

41 Ebd., S. 107; Eichholtz, Krautaktion, S. 271ff. 
gen würde. ${ }^{42}$ Die Versuche, auf einheitlicher Grundlage und nach einheitlichen Richtlinien durchgeführt, umfassten die Messung der Leistung, die Veränderungen des Krankenstandes und der Unfallhäufigkeit. Die Untersuchungsrichtlinien verdeutlichen die Zwangslage und den vollkommenen Objektstatus der Arbeitskräfte. Gleichzeitig versuchen sie, den wissenschaftlichen Anspruch des Vorhabens zu unterstreichen. Es heißt u. a.: „Die Verpflegung der Versuchungspersonen ist von der der Nichtbeteiligten völlig getrennt $\mathrm{zu}$ halten, damit ein Austausch der Nahrungsmittel unterbleibt. Die Ausgabe und der Verzehr der Nahrungsmittel sind genau zu überwachen. Wenn möglich, sind die an der Untersuchung Beteiligten auch unterkunftsmässig getrennt $\mathrm{zu}$ halten und in irgendeiner Form zu kennzeichnen“ (Punkt 5). „Bei Beginn der Zulage ist den beteiligten Ausländern mitzuteilen, dass nach 4 Wochen eine Steigerung ihres Arbeitspensums um einen bestimmten Betrag erwartet wird. [...] Falls innerhalb von 4-6 Wochen die verlangte Leistungssteigerung ausbleibt, ist die Zulage zu entziehen. Die Höhe der verlangten Leistungssteigerung wird von den beteiligten Werken mit dem Kaiser-Wilhelm-Institut vereinbart" (Punkt 8). ${ }^{43}$ Vereinzelt geht die wegen der besseren Ernährung konstatierte Leistungssteigerung mit einem Gewichtsverlust einher. Mit anderen Worten: Die Leistungsbereitschaft übersteigt die Leistungsfähigkeit! Jedoch betont Kraut als allgemeine Tendenz, dass die mit der Gewichtszunahme, durchschnittlich etwa ein kg pro Mann, einhergehende Leistungssteigerung von früher 60 bis 80 Prozent nun auf 80 bis 100 Prozent der deutschen Normalleistung gestiegen sei. Eine Arbeitsintensivierung zwischen 22 und 31 Prozent führe zum Gewichtsstillstand und eine Steigerung von 38 Prozent bereits zum Gewichtsverlust. ${ }^{44}$

Die Rahmenbedingungen, die die Wissenschaftlichkeit seiner Experimente beeinträchtigten, wurden von Kraut durchaus registriert, etwa zu geringe Rationen, Verminderung der Ruhezeiten, Zerstörungen durch Luftangriffe mit häufigen Arbeitsunterbrechungen sowie Materialmangel..$^{45}$ In Folge dessen vermisste er die „frühere(n) Exaktheit“ wie er sie von Laborexperimenten kannte. Aber diese „Störungen“, so sein Zweckoptimismus, hätten keine nachteiligen Folgen für die Ergebnisse, denn „einwandfreie Befunde“ konnten erhoben werden. ${ }^{46}$ Sie würden auch nicht dadurch beeinträchtigt, dass das Untersuchungssample sich um 300 Personen durch Flucht, Selbstverstümmelung und

42 Raehlmann, Arbeitswissenschaft Nationalsozialismus, S. 280.

43 Bundesarchiv Koblenz (künftig: BArch Koblenz) 1198, Nr. 4, Bl. 2 ff.

44 Raehlmann, Arbeitswissenschaft Nationalsozialismus, S. 121.

45 Ebd.

46 BArch Koblenz N 1198, Nr. 33, Bl. 3. 
Sabotage verringerte. ${ }^{47}$ Unter Gesichtspunkten statistischer Auswertung mögen die erwähnten Sachverhalte $\mathrm{zu}$ vernachlässigen sein. Mitnichten $\mathrm{zu}$ vernachlässigen sind allerdings die forschungsethischen Probleme. Die Untersuchungsberichte klammern die Arbeits- und Lebenslage der Untersuchungspersonen aus. Sie abstrahieren zumeist von der Realität menschlicher Not, Verelendung und Zerstörung. Kraut war die katastrophale Lage der Häftlinge durch Besuche seines Assistenten in den Lagern und Betrieben wohl bekannt, zumal er auch eingestehen musste, dass einige Häftlinge erst „aufgefüttert“ werden mussten, um an den Untersuchungen überhaupt teilnehmen zu können. Sein Mitarbeiter berichtete über eine Reise Ende 1944 Folgendes: Die russischen Ärzte unter den Häftlingen beklagen die „dünner gewordene Ernährung“, verweisen auf „eine Häufung grippöser Erkrankungen“ u. a. auf Grund fehlender Decken, ferner auf „Magengeschwüre, eitrig entzündete Hauterkrankungen, Grippe, Tbc“ und Furunkulose wegen geringer Waschmittel, vollständiges „Fehlen von Seife, Handtüchern und einer nur aus Resten bestehenden Unterwäsche“ sowie auf fehlende Strümpfe. ${ }^{48}$ Das ganze menschliche Leid scheint in dem zynisch formulierten Satz auf: „Im S-Lager sammelt sich der Abschaum aller russ. Läger, die Leute sind größtenteils bei ihrer Ankunft im Lager in einem sehr reduzierten Körper- und Ernährungszustand“. ${ }^{49}$

In der Nachkriegszeit beschäftigten sich die KWI-Wissenschaftler weiterhin mit dem Zusammenhang von Ernährung und Leistung, ohne jedoch ihre voraus gegangenen Forschungen zu erwähnen. Der Institutsleiter Lehmann schrieb: „Aktuelle, zeitbedingte Aufgaben erwuchsen dem Institut in nicht zu bewältigender Fülle aus dem unfreiwilligen Experiment der Unterernährung eines ganzen Volkes und ihrer Auswirkung auf die industrielle Leistung. Um die einmalige Gelegenheit zur Erforschung dieser Dinge nicht vorübergehen zu lassen, wurde das Schwergewicht der Institutsarbeit bewusst auf die Seite der Ernährungsfragen verschoben“. ${ }^{50}$ Immerhin konnte Lehmann erreichen, „daß die Ernährungssituation der Industriearbeiter erheblich verbessert und damit die Grundlage für den wirtschaftlichen Wiederaufbau für die Industrie gelegt wurde". ${ }^{1}$

47 Raehlmann, Arbeitswissenschaft Nationalsozialismus, S. 4.

48 Ebd., S. 1f.

49 Ebd., S. 5.

50 AGMPG V Abtl. Rep. 4 KWG 1.

51 AGMPG II, 1 A, PA Lehmann. 


\section{Fazit}

Das wissenschaftliche Selbstverständnis des KWI für Arbeitsphysiologie, bis heute durchaus noch weit verbreitet, ist unter Bezug auf die Wissenschaftslehre Max Webers zu diskutieren..$^{52}$ Damit verbindet sich die Erwartung, dass Handeln der KWI-Wissenschaftler in seiner Komplexität erklären zu können. Die „inhumane Entgrenzung ${ }^{\text {"53 } 33}$ ist eben nicht nur durch wissenschaftliche Neugier, berufliche Ambitionen und Anpassung an politische Erwartungen zu deuten, sondern dafür ist meines Erachtens auch das wissenschaftliche Selbstverständnis der Akteure heranzuziehen. Mit ihrem Anspruch auf Grundlagenforschung verbindet sich bei den KWI-Wissenschaftlern die Vorstellung von objektiver, freier und unabhängiger Forschung.

Zur Erinnerung: Beim Prinzip der Wertfreiheit geht es Weber im Wesentlichen um zwei Dinge, um die Forderung nach Werturteilsfreiheit und um das Problem der Wertbeziehung. Mit dem Prinzip der Werturteilsfreiheit verlangt Weber die Trennung von Sein und Sollen. Der Grundsatz der Wertbeziehung besagt, dass es eine voraussetzungslose Wissenschaft nicht gibt. Die subjektiven und damit normativen Erkenntnisinteressen der WissenschaftlerInnen leiten die Auswahl des Untersuchungsthemas, sie weisen der Forschung die Richtung, bestimmen ihre Struktur und prägen ihre Analyse. Das Prinzip der Wertfreiheit wurde und wird bis heute in der Wissenschaft häufig, so auch damals von den Wissenschaftlern des KWI für Arbeitsphysiologie, unter Aussparung des Prinzips der Wertbeziehung, also nur einseitig rezipiert. Damit wird der Illusion Vorschub geleistet, Wissenschaft sei voraussetzungslos, neutral und folglich den politischen und gesellschaftlichen Rahmenbedingungen mit ihren Problemen und Auseinandersetzungen entrückt. Das ist jedoch mitnichten der Fall.

Bei den militärischen Vorhaben war der Kontext von vornherein klar. Die Möglichkeit eines weiteren Krieges zur Korrektur des „Diktats von Versailles“ zeichnete sich bereits mit dem Aufstieg der NS-Bewegung, dem Beginn und im Verlauf der NS-Herrschaft ab. Die weiteren Kriegspläne hatten die Weltherrschaft im Visier. Aber auch bei den Ernähungsexperimenten zeigte sich, dass in allen Phasen des Forschungsprozesses, d.h. in der Phase der Entdeckung, der Begründung und der Verwertung Einflüsse des umfassenden sozialen Kontextes nachweisbar waren. In der die Forschung abschließenden Phase finden sich in den Dokumenten Vorschläge zur Verwertung der Ergebnisse im Sinne einer

52 Weber, Wertfreiheit, S. $263 f f$.

53 Heim, Kalorien, S. 256. 
Verbesserung der Arbeitsbedingungen für die Zwangsarbeiter, so Arbeitspausen bei Bandarbeit, keine pauschale Verlängerung der Arbeitszeit angesichts der Ernährungslage und generell eine bessere Ernährung. Jedoch hatten diese Vorschläge keine Umsetzungschance. Sie waren reichlich illusionär angesichts der überaus lebensbedrohenden Gesamtlage der Zwangsarbeiter und des rassistischen und imperialistischen Leitbildes des Nationalsozialismus.

Das naturwissenschaftliche Selbstverständnis der KWI-Forscher leistet dieser Selbsttäuschung Vorschub. Zudem geht damit die Überzeugung einher, Laborexperimente seien gegenüber der Feldforschung wissenschaftlich überlegen. Bereits Weber merkte ja dazu schon an, dass Ergebnisse aus der Laborwelt nicht auf die reale Arbeitswelt übertragen werden können. Die Ernährungsuntersuchungen und die Gasschutzforschung waren zwar keine Laborexperimente. Gleichwohl wurden sie als Experimente angesehen, denn der Anspruch war, den Untersuchungsverlauf wie im Labor „total“ zu kontrollieren bzw. kontrollieren zu können. Da das gesamte Leben - bestenfalls Überleben - der Teilnehmer sich in „totalen Institutionen“ wie KZ-Lagern und Militär/Kasernen abspielte, schien die „totale“ Überwachung möglich. ${ }^{54}$ Gemäß diesem Wissenschafts- und Methodenverständnis setzte Kraut in der Nachkriegszeit seine Untersuchungen in weiteren „totalen Institutionen“ wie in Gefängnissen und in Waisenhäusern fort. ${ }^{55}$ Noch in den siebziger Jahren, als im Zusammenhang mit dem staatlichen Forschungs- und Aktionsprogramm „Humanisierung der Arbeit“ eine heftige Kontroverse zwischen Arbeitsmedizin/Ergonomie und Soziologie um das Profil der Arbeitwissenschaft ausgetragen wurde, waren diese Unterschiede im methodischen Vorgehen - Laborexperimente versus Feldforschung - ein herausragender Streitpunkt. ${ }^{56}$ Inzwischen wird zunehmend ein Methodenpluralismus als ein Kennzeichen von Interdisziplinarität akzeptiert. Mit Blick auf die Zusammenarbeit von Sozial- und Naturwissenschaften allgemein ist meines Erachtens jedoch davon auszugehen, dass Spannungen und Konflikte über das methodische Vorgehen, ja letztlich über das wissenschaftliche Selbstverständnis nach wie vor existieren. Es bleibt für das System der Wis-

54 Erving Goffman, Asyle. Über die soziale Situation psychiatrischer Patienten und andere Insassen, Frankfurt a.M. 1973; Michel Foucault, Überwachen und Strafen. Die Geburt des Gefängnisses, in: ders., Die Hauptwerke, Frankfurt a.M. 2008, S. 705-1019.

55 Thoms, Nachkriegskarriere, S. 315ff.

56 Raehlmann, Arbeitswissenschaft Weimarer Republik, S. 9ff.; dies., Arbeitswissenschaft im Spannungsfeld von systemischer Rationalisierung und Globalisierung, in: Aida Bosch et al. (Hrsg.), Sozialwissenschaftliche Forschung und Praxis. Interdisziplinäre Sichtweisen, Wiesbaden 1999, S. 181-209; Irene Raehlmann/Walter Volpert, Geschichte und Richtungen der Arbeitswissenschaft, in: Holger Luczak/Walter Volpert (Hrsg.), Handbuch Arbeitswissenschaft, Stuttgart 1997, S. 19-25. 
senschaft und für seine Akteure eine erhebliche Herausforderung diesen Pluralismus anzuerkennen.

Die vorgestellten Forschungsergebnisse aus dem KWI für Arbeitsphysiologie bzw. aus der KWG müssten der MPG wohl bekannt sein, zumal die referierten Veröffentlichungen ihnen vermutlich vorliegen. Als Soziologin ist mir das Phänomen des Vergessens bzw. des Wiederentdeckens in der Wissenschaft geläufig. Jüngst habe ich mit Erstaunen eben dieses Phänomen auch in einer MPG-Publikation, worauf ein Artikel in der Süddeutschen Zeitung (SZ) verwies, zur Kenntnis genommen. Mittlerweile haben zahlreiche Untersuchungen der letzten Jahrzehnte unser Wissen über die NS-Zeit ausgeweitet und vertieft. Umso irritierender erscheint nun die Tatsache, dass - laut SZ - Historiker wohl zu Recht beklagen, die MPG gehe „nach Jahren vorbildlicher Aufarbeitung [...] nun leichtfertig mit ihrer NS-Geschichte um “. ${ }^{57}$ In dem Beitrag kritisierte die Autorin Christina Berndt u. a. mit Blick auf das KWI für Arbeitsphysiologie, dass in dem MPG-Wissenschaftsmagazin unter der Rubrik „Rückblende“ über die vorgenannten Forschungen kein Wort zu lesen ist. Es sind, wie die Überschrift ihres SZ-Artikels lautet: „Blinde Flecken“! Berichtet wird zwar über frühere Forschungen, etwa über einen Trainingsplan für einen Waschbrettbauch unter dem Titel „Schwitzen im Dienst der Wissenschaft“ oder über Fahrradfahren unter Alkoholeinfluss ${ }^{58} \mathrm{Zu}$ fragen ist daher: Haben wir es - trotz weiterer, einschlägiger NS-Forschungen - in so kurzer Zeit mit einem einzelnen, zufälligen Vergessen oder schon mit einem allgemeinen, systematischen, erneuten Vergessen und Verdrängen zu tun? Ist ein einmal erreichtes Niveau an Aufklärung immer wieder gefährdet?

57 Christina Berndt, Blinde Flecken. Nach Jahren vorbildlicher Aufarbeitung geht die MaxPlanck-Gesellschaft nun leichtfertig mit ihrer NS-Geschichte um, beklagen Historiker, in: Süddeutsche Zeitung, Nr. 19 vom 25.1.2011, S. 16.

58 Elke Maier, Schwitzen im Dienst der Wissenschaft, in: Max Planck Forschung. Das Wissenschaftsmagazin der Max-Planck-Gesellschaft (2009) 3, S. 96f. 